\title{
Adenovirus type 3 induces platelet activation in vitro
}

\author{
YING-YU JIN ${ }^{1,2}$, XIU-NAN YU ${ }^{2}$, ZHANG-YI QU ${ }^{1}$, AI-AI ZHANG ${ }^{2}$, YU-LING XING ${ }^{2}$, \\ LI-XIN JIANG $^{2}$, LEI SHANG ${ }^{1}$ and YING-CHEN WANG ${ }^{1}$ \\ ${ }^{1}$ Department of Hygienic Microbiology, Public Health College, Harbin Medical University, Harbin, Heilongjiang 150081; \\ ${ }^{2}$ Department of Laboratory Diagnosis, The First Clinical College, Harbin Medical University, \\ Harbin, Heilongjiang 150001, P.R. China
}

Received March 23, 2013; Accepted November 5, 2013

DOI: $10.3892 / \mathrm{mmr} .2013 .1805$

\begin{abstract}
In the present study, we aimed to investigate platelet activation induced by adenovirus type 3 (HAdV3) in vitro. Platelet-rich plasma (PRP) or whole blood was incubated with or without HAdV at various concentrations. Platelet aggregation, platelet counting, fibrinogen and expression of platelet membrane antigens (CD41a and CD62P) were determined following incubation with $\mathrm{HAdV}$ for different periods of time. The results demonstrated that $\mathrm{HAdV}$ at the concentrations of $10^{9}-10^{11} \mathrm{vp} / \mathrm{ml}$ enhanced adenosine diphosphate (ADP) or ristocetin-induced platelet aggregation, however did not alter the platelet count. Infection with HAdVs also reduced fibrinogen level. P-selectin and CD41a appeared rapidly on the surface after platelets were incubated with HAdVs in vitro for $30 \mathrm{~min}$. In conclusion, HAdVs may induce activation of platelets and lead to a pre-thrombotic state of peripheral blood. This finding may aid in the development of measures to prevent severe HAdV infection.
\end{abstract}

\section{Introduction}

Platelets are essential for the maintenance of hemostasis through adhesion, aggregation/cohesion and the release of thrombin and cytokines. Platelet hyperfunction may be involved in various abnormalities and diseases, including thrombosis, atherosclerosis, tumor progression/metastasis, stroke and myocardial infarction induced by arterial thrombosis (1). In addition to thrombosis, platelets are also a pivotal player in the host defense response (2).

Correspondence to: Professor Zhangyi Qu, Department of Hygienic Microbiology, Public Health College, Harbin Medical University, 157 Baojian Road, Harbin, Heilongjiang 150081, P.R. China E-mail: jinyingyustar@163.com

Abbreviations: PRP, platelet-rich plasma; RGD, arginine-glycine-aspartic acid; ADP, adenosine diphosphate; HAdV3, human adenovirus type 3

Key words: adenovirus, platelet, CD62P, CD41a, fibrinogen, adenovirus type 3
Virus infection is able to stimulate a series of platelet responses, including platelet binding and engulfment. Furthermore, activated platelets express P-selectin, which mediates the elimination of viruses by macrophages $(3,4)$. Following activation, platelets release granular contents, which contain biologically active peptides that target the immune system through RANTES, IL-1 $\beta$, MCP-1, PF-4 and PAF pathways (5). Thus, platelets are essential in regulating thrombosis and inflammation.

Human adenovirus (HAdV) is a common cause of human acute respiratory diseases. HAdVs also cause conjunctivitis, gastrointestinal and urinary tract infections and occasionally encephalitis. Platelets are important in the innate immune response against $\mathrm{HAdV}$ infection. Integrin $\alpha \operatorname{IIb} \beta 3$, which recognizes arginine-glycine-aspartic acid (RGD) sequences, is expressed on the surface of platelets (6). RGD sequences are also present in the penton base of HAdV. HAdV is able to bind to macrophage cells, which express $\alpha \operatorname{IIb} \beta 3$, suggesting the possibility of binding between the RGD sequences of HAdVs and $\alpha I I b \beta 3$ on the surface of platelets (7). Infection of HAdV into the cells involves RGD sequence mediated adhesion to the cells and virus type-dependent entry into the cells. The interaction between the virus and $\alpha \operatorname{IIb} \beta 3$ leads to the activation of platelets and is required for the internalization of $\operatorname{HAdV}(8,9)$. However, the effect of HAdV on platelet activation is controversial: Eggerman et al demonstrated that an HAdV vector had no effect on platelet aggregation (10), while later Othman et al demonstrated that an HAdV vector did induce platelet activation (3). Thus, the platelet response to HAdV infection requires further examination.

HAdV type 3 (HAdV3) is the most common type of clinical HAdV infection (11). HAdV3 infection is particularly severe among young children and immunocompromised adults (12). Understanding the platelet response to HAdV infection may aid the development of measures to prevent severe HAdV infection. In the present study, we studied platelet-HAdV interactions in vitro following the establishment of a whole blood and platelet-rich plasma model infected with HAdV3. We demonstrated that HAdV at a certain concentration range may potentiate ADP and ristocetin-induced platelet aggregation. A rapid increase of CD41a and CD62P expression following incubation with HAdV was also observed. 


\section{Materials and methods}

Virus purification. HAdV3 was obtained from ATCC (Manassas, VA, USA). The virus was propagated in A549 cells and was harvested when the cytopathic effect (CPE) reached $>95 \%$ by freezing $\left(-80^{\circ} \mathrm{C}, 10 \mathrm{~min}\right)$ and thawing (room temperature) the cell culture flasks three times. The supernatant was stored at $-80^{\circ} \mathrm{C}$ following a low speed centrifugation (3000 x g for $5 \mathrm{~min}$ ) (13). The purification of the virus was performed using the VirTrap ${ }^{\text {TM }}$ HAdV purification kit (Biomiga, San Diego, CA, USA). Purified virus was quantified by spectrophotometric measurement of the optical density at OD260 and recorded as virus particles per milliliter (vp/ml).

Blood collection and preparation of platelets. Ten blood donors (aged from 24 to 47 years) were selected based on the following criteria: healthy volunteers with normal platelet aggregation; parameters of coagulation and other whole blood indicators were normal and no symptoms of flu were found four weeks prior to blood withdrawal. Written informed consent was obtained from the donors and the study was approved by the Ethics Committee of the first clinical college of Harbin Medical University (Harbin, China). Sodium citrate (109 $\mathrm{mM}$ ) was added to the whole blood at the ratio of 1:9 to prevent platelet clumping prior to centrifuging at $800 \mathrm{x} \mathrm{g}$ for $7 \mathrm{~min}$. The supernatant was platelet-rich plasma (PRP) and the platelet count was adjusted to $160-380 \times 10^{9} / 1$ using corresponding platelet-free plasma, which was prepared by depleting platelets with centrifugation at $3000 \mathrm{x}$ g for $10 \mathrm{~min}$. At least 5 replicates of each of the following experiments were performed.

Measurement of platelet aggregation, platelet counting and fibrinogen. PRP $(500 \mu \mathrm{l})$ was incubated with $10 \mu \mathrm{l}$ of HAdV at the concentration range of $10^{9}-10^{11} \mathrm{vp} / \mathrm{ml}$ at $37^{\circ} \mathrm{C}$ in vitro for $1,30,40,50,60,70,80,90,100$ and $130 \mathrm{~min}$, respectively. Adenosine diphosphate (ADP; $10 \mu \mathrm{M})$ and ristocetin $(1.0 \mathrm{~g} / \mathrm{l})$ were used as inducers of platelet aggregation. The inducer $(2.5 \mu \mathrm{l})$ was added to PRP for each experiment. The rate of platelet aggregation was measured by a 560CA Aggregometer (Chrono-Log, Havertown, PA, USA). Following incubation of anticoagulated whole blood with $2 \mu \mathrm{l}\left(10^{10} \mathrm{vp} / \mathrm{ml}\right)$ of the virus, the number of platelets was counted by a MEK-6318 hematology analyzer (Nihon Kohden, Tokyo, Japan). Fibrinogen in platelet-depleted plasma, which was obtained following centrifugation, was analyzed by a Sysmex-1500 automated coagulation analyzer (Sysmex Corporation, Kobe, Japan). Corresponding amounts of PRP or anticoagulated whole blood served as controls.

Expression of P-selectin and CD41a in platelets. P-selectin is a surface marker of activated platelets. To measure P-selectin expression, $500 \mu 1$ of PRP was incubated with $10 \mu \mathrm{l}$ of $10^{10} \mathrm{vp} / \mathrm{ml} \mathrm{HAdV}$ at $37^{\circ} \mathrm{C}$ in vitro for 1 and $30 \mathrm{~min}$, respectively, prior to staining with $\mathrm{PE}$ conjugated antihuman CD62P (P-selectin; Becton-Dickinson, Inc., Franklin Lakes, NJ, USA) and FITC conjugated anti-human CD41a (Becton-Dickinson, Inc.) antibodies. Expression of P-selectin and CD41a was determined by Canto II flow cytometry
(Becton-Dickinson, Inc.). PRP with virus-free buffer solution served as the control.

Statistical analysis. Continuous variables with a normal distribution were expressed as the mean \pm SEM. The platelet counts, fibrinogen and platelet aggregation induced by ADP or ristocetin, which were measured at different time points and at different concentrations, were subjected to ANOVA analysis. The Greenhouse-Geisser correction was applied to all the data due to the violation of sphericity. Independent sample t-tests were applied to compare the rate of platelet aggregation, CD41a and CD62P between the control and virus groups. All data were processed by SPSS 18.0 statistical software and the conditions were as follows: two-sided test, $\alpha=0.05$ as the test level and $\mathrm{P}<0.05$ was considered to indicate a statistically significant difference.

\section{Results}

HAdV potentiates ADP-induced platelet aggregation. ADP-induced platelet aggregation was measured by the Chrono-Log Aggregometer (Fig. 1). Pre-incubation of PRP with $10^{9}-10^{11} \mathrm{vp} / \mathrm{ml}$ of $\mathrm{HAdV}$ for up to $130 \mathrm{~min}$ caused the rate of ADP-induced platelet aggregation to markedly increase (Fig. 1). However, the platelet aggregation was not affected by the length of incubation time with the HAdV (Fig. 1) and was not dose dependent.

Lower concentrations of HAdV $\left(10^{7}-10^{8} \mathrm{vp} / \mathrm{ml}\right)$ demonstrated no effect (Fig. 2), however HAdV concentrations of $10^{9}-10^{11} \mathrm{vp} / \mathrm{ml}$ significantly enhanced ADP-induced platelet aggregation.

$H A d V$ potentiates ristocetin-induced platelet aggregation. Similar to ADP-induced platelet aggregation, pre-incubation of PRP with $10^{9}-10^{11} \mathrm{vp} / \mathrm{ml}$ of HAdV significantly increased ristocetin-induced platelet aggregation (Fig. 3). However, there was no difference among the three dosages $\left(10^{9}, 10^{10}\right.$ and $10^{11} \mathrm{vp} / \mathrm{ml}$ ) or incubation time (Fig. 3).

HAdV did not alter the platelet count or fibrinogen. HAdV $\left(10^{10} \mathrm{vp} / \mathrm{ml}\right)$ was incubated with whole blood for the indicated time periods (1-130 min) prior to platelet counting. Variance analysis demonstrated no statistical significance in platelet counts among all groups $(\mathrm{df}=1, \mathrm{~F}=0.15, \mathrm{P}=0.74)$ or among different time points $(\mathrm{df}=1.88, \mathrm{~F}=1.25, \mathrm{P}=0.38)$.

$10^{10} \mathrm{vp} / \mathrm{ml}$ of HAdV was incubated with whole blood for the indicated time periods (1-130 $\mathrm{min})$ prior to fibrinogen measurement. Variance analysis of fibrinogen suggested no significance among groups $(\mathrm{df}=1, \mathrm{~F}=7.74, \mathrm{P}=0.11)$ or among time points $(\mathrm{df}=1.37, \mathrm{~F}=0.94, \mathrm{P}=0.45)$.

We demonstrated that incubation of whole blood with $10^{10} \mathrm{vp} / \mathrm{ml}$ of HAdV did not alter platelet count and fibrinogen in anticoagulated whole blood. There was no significant difference in platelet count and fibrinogen among the control and virus groups at different time points $(\mathrm{P}>0.05$; data not shown).

HAdV induced P-selectin and CD4la expression on platelets. Pre-incubation of PRP with HAdV for 30 min increased the percentage of $\mathrm{CD} 62 \mathrm{P}$-positive platelets from $0.38 \pm 0.07$ to 


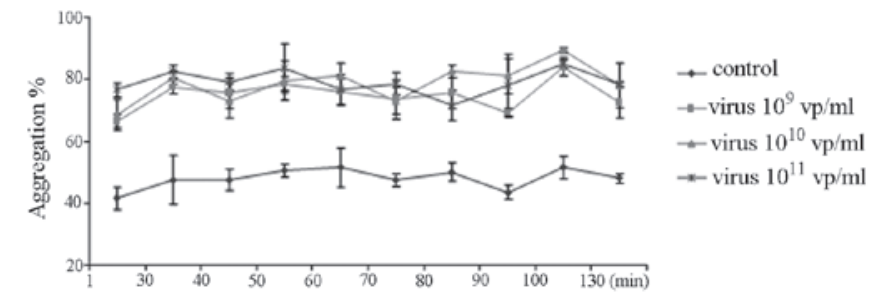

Figure 1. Adenovirus potentiates ADP-induced platelet aggregation. PRP was incubated with adenovirus at the range of $10^{9}-10^{11} \mathrm{vp} / \mathrm{ml}$ at $37^{\circ} \mathrm{C}$ for 1-130 $\mathrm{min}$. The rate of platelet aggregation was measured following incubation. ADP, adenosine diphosphate; PRP, platelet-rich plasma.

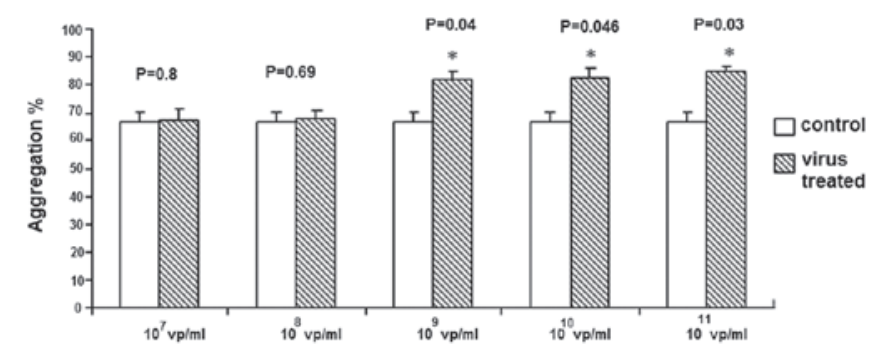

Figure 2. Adenovirus $\left(10^{9}-10^{11}\right)$ potentiates ADP-induced platelet aggregation. PRP was incubated with adenovirus at the range of $10^{7}-10^{11} \mathrm{vp} / \mathrm{ml}$ at $37^{\circ} \mathrm{C}$ for $90 \mathrm{~min}$. The rate of platelet aggregation was measured following incubation. ${ }^{*} \mathrm{P}<0.05$, compared with the control group. ADP, adenosine diphosphate; PRP, platelet-rich plasma.

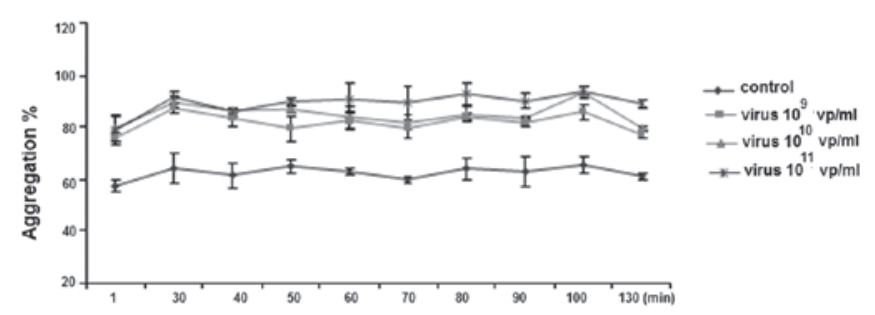

Figure 3. Adenovirus potentiates ristocetin-induced platelet aggregation. PRP was incubated with adenovirus at the range of $10^{9}-10^{11} \mathrm{vp} / \mathrm{ml}$ at $37^{\circ} \mathrm{C}$ for 1-130 $\mathrm{min}$. The rate of platelet aggregation was measured following incubation. PRP, platelet-rich plasma.

$0.70 \pm 0.02(\mathrm{P}=0.03)$, indicating activation of the platelets. Similarly, 30 min of treatment with HAdV increased the mean channel fluorescence intensity of CD41a surface staining from $247.6 \pm 105.7$ to $382.3 \pm 71.7(\mathrm{P}=0.04)$ compared with the control group (Fig. 4).

\section{Discussion}

HAdV is the second most common pathogen, which causes adult community-acquired pneumonia (14). HAdV3 is one of the most prevalent serotypes detected globally and its variants have been associated with outbreaks of severe disease (12). Recently, HAdV3 infection demonstrated an increasing trend (11). However, the majority of studies are focused on the interaction between platelets and HAdV type 5 (often used as a gene vector), whereas studies on the wild-type HAdV are scattered $(6,9)$. In particular, platelet function during $\mathrm{HAdV}$ infection is unclear (15). This study provided evidence that
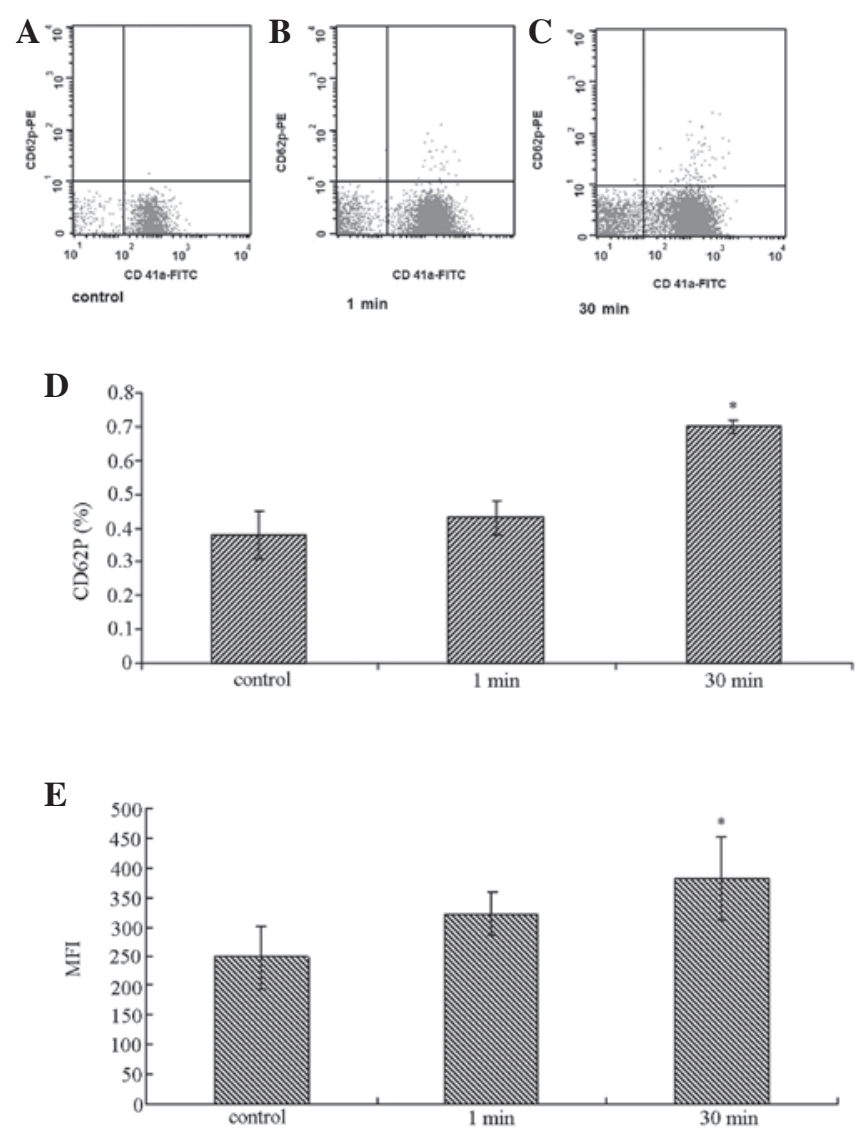

Figure 4. Detection of CD62P and CD41a on the surface of platelets by flow cytometry. PRP was incubated with $10 \mu 1$ of $10^{10} \mathrm{vp} / \mathrm{ml}$ adenovirus at $37^{\circ} \mathrm{C}$ in vitro for 1 and $30 \mathrm{~min}$, respectively, prior to staining with PE-conjugated anti-human CD62P (P-selectin; BD Biosciences, Franklin Lakes, NJ, USA) and FITC-conjugated anti-human CD41a (Becton-Dickinson, Inc., Franklin Lakes, NJ, USA) antibodies. PRP with virus-free buffer solution served as the control. ${ }^{*} \mathrm{P}<0.05$, compared with the 1 min control group. PRP, plateletrich plasma; MFI, mean fluorescence intensity.

HAdV infection may activate platelets and increase platelet aggregation.

The platelet aggregation test is routinely used to detect the state of platelet activation in the blood. $\alpha \operatorname{IIb} \beta 3$ integrin on platelets is important in platelet aggregation and thrombus formation. The major platelet receptor, $\alpha \operatorname{IIb} \beta 3$ integrin is inactive in quiescent cells, however inducers, including ADP and thrombin or platelet activators, may lead to its persistent activation and binding to fibrinogen and other ligands $(16,17)$. Thus, a large number of micro-organisms are able to enter into the cells mediated by integrin (18). We demonstrated that the $\mathrm{HAdV}$ at a certain concentration range $\left(10^{9}-10^{11} \mathrm{vp} / \mathrm{ml}\right)$ is able to induce platelet aggregation. It is likely that the binding of the RGD sequence of HAdV and $\alpha \mathrm{IIb} \beta 3$ integrin on platelets leads to the activation of platelets. $\alpha \operatorname{IIb} \beta 3$ on the activated platelet surface may also bind to fibrinogen to facilitate the aggregation of platelets (19).

In the present study, the rate of platelet aggregation did not change with the length of incubation time (up to $130 \mathrm{~min}$ ). This may be explained by the short incubation periods we selected since it is advised to finish platelet aggregation tests in vitro within $2 \mathrm{~h}$. However, in vivo experiments have demonstrated that HAdV type 5 induces platelet activation $6 \mathrm{~h}$ after the virus is injected into the body (9). 
Among various concentrations of HAdV we examined, only $10^{9} \mathrm{vp} / \mathrm{ml}$ and above potentiated the significant aggregation of platelets, suggesting that platelet activation is concentration dependent. It is possible that less HAdV binds to glycoprotein (GP) IIb-IIIa ( $\alpha$ IIb $\beta 3$-integrin) at lower concentrations. Thus, certain amounts of activated integrin may be required to form the bridge among platelets and HAdVs. Although no direct evidence suggests the expression of $\alpha \operatorname{IIb} \beta 3$ integrin is able to directly induce platelet aggregation, pharmacokinetic studies of an $\alpha \operatorname{IIb} \beta 3$ integrin antagonist demonstrate a marked correlation between platelet aggregation and the quantity of free platelet GP IIb-IIIa receptors (20).

Recently, studies have demonstrated that the injection of HAdV 3 causes thrombocytopenia in mice and clinical studies have also demonstrated a decreased platelet count following infection with HAdV in humans $(21,22)$. However, our study demonstrated a slight decrease in platelet counts without statistical significance following HAdV infection. This may be explained by the activation of the anti-inflammatory defense system stimulated by platelets infected with HAdV, which subsequently activate phagocytosis by monocytes/macrophages to engulf HAdV infected platelets $(9,19)$. However, there is no such mechanism in vitro, which may explain the insignificant decrease of platelets.

In order to confirm the role of HAdV in platelet activation, we next measured the CD62P expression on the membrane of platelets. P-selectin is a cell adhesion molecule expressed in platelets and endothelial cells. It is usually stored in secretory granules and rapidly expressed on the plasma membrane following the activation of platelets (23). P-selectin plays two major roles: firstly, P-selectin re-distributes on the surface of activated endothelial cells (to mediate the rolling of the leukocyte) during inflammation $(24,25)$; secondly, P-selectin expression on activated platelets (in the thrombus) enhances leukocyte recruitment in the process of thrombosis (26). Thus, P-selectin expressed on platelets is involved in the processes of inflammation, thrombosis and coagulation and may serve as an indicator for the hypercoagulable state in vivo $(26,27)$. In the present study, we observed rapid exposure of P-selectin on the platelet surface following the addition of HAdV, suggesting that the interaction between HAdV and platelets leads to platelet activation. In addition, our in vitro data demonstrated a significantly increased expression of CD41a on the surface of platelets incubated with HAdV but not on the resting platelets. Since CD41a is important in platelet aggregation (19), our observation indicated possible platelet aggregation induced by the HAdV.

Antiplatelet therapy reduces the inflammatory response by inhibiting platelet activation and aggregation (28). Our results suggested that HAdV3 may induce the activation of platelets and lead to a prothrombotic state. Our results may assist in the development of measures to prevent severe HAdV infection.

\section{Acknowledgements}

This study is supported by the National Natural Science Foundation of China (grant no. 30771909 and grant no. 81172725$)$.

\section{References}

1. Picker SM: In-vitro assessment of platelet function. Transfus Apher Sci 44: 305-319, 2011.

2. Klinger MH and Jelkmann W: Role of blood platelets in infection and inflammation. J Interferon Cytokine Res 22: 913-922, 2002.

3. Othman M, Labelle A, Mazzetti I, Elbatarny HS and Lillicrap D: Adenovirus-induced thrombocytopenia: the role of von Willebrand factor and P-selectin in mediating accelerated platelet clearance. Blood 109: 2832-2839, 2007.

4. Youssefian T, Drouin A, Massé JM, Guichard J and Cramer EM: Host defense role of platelets: engulfment of HIV and Staphylococcus aureus occurs in a specific subcellular compartment and is enhanced by platelet activation. Blood 99: 4021-4029, 2002

5. Senzel L, Gnatenko DV and Bahou WF: The platelet proteome. Curr Opin Hematol 16: 329-333, 2009.

6. Zhang Y and Bergelson JM: Adenovirus receptors. J Virol 79: 12125-12131, 2005.

7. Faraday N, Rade JJ, Johns DC, Khetawat G, Noga SJ, DiPersio JF, Jin Y, Nichol JL, Haug JS and Bray PF: Ex vivo cultured megakaryocytes express functional glycoprotein IIb-IIIa receptors and are capable of adenovirus-mediated transgene expression. Blood 94: 4084-4092, 1999.

8. Triantafilou K, Triantafilou M, Takada Y and Fernandez N: Human parechovirus 1 utilizes integrins alphavbeta3 and alphavbeta1 as receptors. J Virol 74: 5856-5862, 2000.

9. Stone D, Liu Y, Shayakhmetov D, Li ZY, Ni S and Lieber A: Adenovirus-platelet interaction in blood causes virus sequestration to the reticuloendothelial system of the liver. J Virol 81: 4866-4871, 2007.

10. Eggerman TL, Mondoro TH, Lozier JN and Vostal JG: Adenoviral vectors do not induce, inhibit, or potentiate human platelet aggregation. Hum Gene Ther 13: 125-128, 2002.

11. Gray GC, McCarthy T, Lebeck MG, et al: Genotype prevalence and risk factors for severe clinical adenovirus infection, United States 2004-2006. Clin Infect Dis 45: 1120-1131, 2007.

12. Lebeck MG, McCarthy TA, Capuano AW, Schnurr DP, Landry ML, Setterquist SF, Heil GL, Kilic S and Gray GC: Emergent US adenovirus 3 strains associated with an epidemic and serious disease. J Clin Virol 46: 331-336, 2009.

13. Shang L, Qu Z, Sun L, Wang Y, Liu F, Wang S, Gao H and Jiang F: Astragaloside IV inhibits adenovirus replication and apoptosis in A549 cells in vitro. J Pharm Pharmacol 63: 688-694, 2011.

14. Angeles Marcos M, Camps M, Pumarola T, Antonio Martinez J, Martinez E, Mensa J, Garcia E, Peñarroja G, Dambrava P, Casas I, Jiménez de Anta MT and Torres A: The role of viruses in the aetiology of community-acquired pneumonia in adults Antivir Ther 11: 351-359, 2006.

15. Maurice A, Marchand-Arvier M, Edert D, Le Faou A, Gondrexon $G$ and Vigneron $C$ : The virucidal effect of platelet concentrates: preliminary study and first conclusions. Platelets 13: 219-222, 2002.

16. Basani RB, French DL, Vilaire G, Brown DL, Chen F, Coller BS, Derrick JM, Gartner TK, Bennett JS and Poncz M: A naturally occurring mutation near the amino terminus of alphaIIb defines a new region involved in ligand binding to alphaIIbbeta3. Blood 95: 180-188, 2000.

17. Shimaoka M and Springer TA: Therapeutic antagonists and conformational regulation of integrin function. Nat Rev Drug Discov 2: 703-716, 2003.

18. Plow EF, Haas TA, Zhang L, Loftus J and Smith JW: Ligand binding to integrins. J Biol Chem 275: 21785-21788, 2000.

19. Yakushkin VV, Zyuryaev IT, Khaspekova SG, Sirotkina OV, Ruda MY and Mazurov AV: Glycoprotein IIb-IIIa content and platelet aggregation in healthy volunteers and patients with acute coronary syndrome. Platelets 22: 243-251, 2011.

20. Mazurov AV, Pevzner DV, Antonova OA, Byzova TV, Khaspekova SG, Semenov AV, Vlasik TN, Samko AN, Staroverov II and Ruda MY: Safety, inhibition of platelet aggregation and pharmacokinetics of Fab'2 fragments of the anti-glycoprotein IIb-IIIa monoclonal antibody FRaMon in high-risk coronary angioplasty. Platelets 13: 465-477, 2002.

21. Appledorn DM, Kiang A, McBride A, Jiang H, Seregin S, Scott JM, Stringer R, Kousa Y, Hoban M, Frank MM and Amalfitano A: Wild-type adenoviruses from groups A-F evoke unique innate immune responses, of which HAd3 and SAd23 are partially complement dependent. Gene Ther 15: 885-901, 2008. 
22. Klinger JR, Sanchez MP, Curtin LA, Durkin M and Matyas B: Multiple cases of life-threatening adenovirus pneumonia in a mental health care center. Am J Respir Crit Care Med 157: 645-649, 1998.

23. McEver RP, Beckstead JH, Moore KL, Marshall-Carlson L and Bainton DF: GMP-140, a platelet alpha-granule membrane protein, is also synthesized by vascular endothelial cells and is localized in Weibel-Palade bodies. J Clin Invest 84: 92-99, 1989.

24. Denis CV, André P, Saffaripour S and Wagner DD: Defect in regulated secretion of P-selectin affects leukocyte recruitment in von Willebrand factor-deficient mice. Proc Natl Acad Sci USA 98: 4072-4077, 2001.

25. Sundd P, Pospieszalska MK, Cheung LS, Konstantopoulos K and Ley K: Biomechanics of leukocyte rolling. Biorheology 48: 1-35, 2011.
26. Palabrica T,Lobb R, Furie BC, Aronovitz M,Benjamin C, Hsu YM, Sajer SA and Furie B: Leukocyte accumulation promoting fibrin deposition is mediated in vivo by P-selectin on adherent platelets. Nature 359: 848-851, 1992.

27. Blann AD, Nadar SK and Lip GY: The adhesion molecule P-selectin and cardiovascular disease. Eur Heart J 24: 2166-2179, 2003.

28. Iannacone M, Sitia G, Narvaiza I, Ruggeri ZM and Guidotti LG: Antiplatelet drug therapy moderates immune-mediated liver disease and inhibits viral clearance in mice infected with a replication-deficient adenovirus. Clin Vaccine Immunol 14: 1532$1535,2007$. 